\title{
Die Errichtung einer Europäischen Finanzmarktunion: Ein Fall von funktionalem spillover
}

\begin{abstract}
Schwächen im Aufbau der Wirtschafts- und Währungsunion (WWU) sind im Verlauf der Finanzkrise zu Tage getreten und führten zu einer vertieften Europäischen Integration. Relativ zügige und bemerkenswerte Integrationsschritte wurden als Antwort auf die Krise unternommen, um unter anderem Schwächen im Bankensystem und in den öffentlichen Finanzen der EU-Mitgliedstaaten zu begegnen. In einigen Fällen war der Handlungsbedarf besonders hoch, da die Mitgliedsstaaten in einem Teufelskreis, dem sogenannten sovereign-bank nexus, gefangen waren, der die Finanzstabilität in der WWU gefährdete. Besonders im Bereich der Finanzaufsicht zeigten sich nationale Behörden schlecht positioniert, um der weiterschreitenden Krise zu begegnen. Die anfänglichen Hilfsaktionen wurden später durch tiefer greifende institutionelle Reformen ergänzt, die zu einer tieferen Integration führten. Dieser Beitrag erklärt diese Entwicklungen aus einer neo-funktionalistischen Perspektive und identifiziert insbesondere das Konzept des funktionalen spillover als eine treibende Kraft dieses Prozesses. Die Krise verstärkte den funktionalen spillover zwischen den verschiedenen Politikbereichen der WWU. Sie wirkte als Auslöser für das zutage Treten zugrundeliegender funktionaler Dissonanzen, welche zur vertieften Integration führten. Wir schlussfolgern, dass dieser Prozess weitere Integrationsschritte nach sich ziehen kann, bis der funktionale Druck entsprechend abgebaut wurde.
\end{abstract}

\section{Einleitung ${ }^{1}$}

Die im Jahr 2007 einsetzende Finanzkrise, die sich in manchen EU-Mitgliedstaaten zu einer Staatsschuldenkrise weiterentwickelte, hat bereits bestehende Schwächen bei der Tragfähigkeit der globalen Finanzarchitektur und insbesondere der Steuerungsfähigkeit des EU-Binnenmarktes offenbart. Schon in der ersten Krisenphase machte der Zusammenbruch von Lehmann Brothers im September 2008 staatliche Interventionen zur Unterstützung des Finanzsystems insbesondere in den Industriestaaten erforderlich. Darüber hinaus haben diese Entwicklungen in Europa strukturelle Schwächen in der Steuerungsfähigkeit des EU-Finanzsektors und des Euro-

1 Die Autoren möchten Johannes Lindner, Benjamin Vonessen und den zwei anonymen Gutachtern der ZIB für ihre hilfreichen Kommentare und Anregungen danken. Weiterer Dank gebührt Lisa Hehnke und Dennis Voll für die exzellente Forschungsassistenz. Die Autoren bleiben verantwortlich für etwaige Fehler und Auslassungen, wobei Entwicklungen bis Ende Februar 2013 berücksichtigt wurden. Die Ansichten, die in diesem Beitrag zum Ausdruck gebracht werden, sind die der Autoren und repräsentieren nicht notwendigerweise die Ansichten der Europäischen Zentralbank. 
raums offen gelegt. Die schon vor der Krise existente öffentliche und/oder private Verschuldung brachte im Verlauf der Krise die zunehmende gegenseitige Abhängigkeit der Banken und Staatsfinanzen (der sogenannte sovereign-bank nexus), die Schwächen des bestehenden aufsichtlichen Rahmens in der EU, die unzureichende Disziplinierung des Finanzsystems durch marktwirtschaftliche Kontrollmechanismen sowie die Gefahr einer gegenseitigen Ansteckung ins öffentliche Bewusstsein. Die Begrenzung einer sich selbst verstärkenden negativen Dynamik, Maßnahmen zur nachhaltigen Stabilisierung der Finanzmärkte sowie eine Verbesserung des institutionellen Rahmens zur Krisenprävention und -bewältigung sind zentrale Herausforderungen auf EU-Ebene. ${ }^{2}$

In der EU wurden verschiedene Schritte unternommen, um diesen Herausforderungen zu begegnen. Die zwischenstaatliche Solidarität wurde durch neue Institutionen verstärkt, die auch zum Aufbrechen des sovereign-bank nexus beitrugen. Darüber hinaus wurden neue europäische Aufsichtsbehörden zur verbesserten Überwachung des Finanzsektors etabliert. Konkrete Schritte für einen einheitlichen Überwachungsmechanismus unter Einbeziehung der Europäischen Zentralbank (EZB) finden derzeit in den Eurostaaten statt. Der systemweiten Dimension von Finanzstabilität wurde u.a. durch die Etablierung des Europäischen Ausschusses für Systemrisiken (ESRB) Rechnung getragen. Dieser Beitrag beschreibt diesen Reformprozess und argumentiert, dass dieser in groben Zügen einer neo-funktionalistischen Integrationslogik folgt: ${ }^{3}$ Nach Einführung der Gemeinschaftswährung entwickelten sich funktionale Diskrepanzen zwischen supranationaler Währungspolitik und integrierten Finanzmärkten auf der einen Seite und intergouvernementaler Fiskalpolitik und nationalen Aufsichtsbehörden auf der anderen Seite. Diese Dissonanzen haben Krisen begünstigt, die dann im Prozess ihrer Bewältigung potenzierte funktionale Sachzwänge nach sich zogen, und letztendlich die notwendigen Integrationsschritte auslösten.

Der Artikel ist wie folgt aufgebaut: Abschnitt 2 fasst die von der EU und den Eurostaaten als Reaktion auf die Krise getroffenen Maßnahmen in verschiedenen Politikfeldern zusammen. Aufbauend hierauf werden in Abschnitt 3 die europäischen Reformen in der Bankenüberwachung im Detail analysiert, und die Dynamiken hin zu einer vertieften Integration beschrieben. Abschnitt 4 dient der Darstellung unseres theoretischen Grundgerüsts. Dabei beziehen wir uns auf einige Grundannahmen des Neofunktionalismus und stellen das Konzept des functional spillover (in etwas überarbeiteter und erweiterter Form) in den Vordergrund. Auf dieses Konzept stützt

2 Einen Überblick zum Krisenverlauf und insbesondere des Zusammenspiels von ökonomischen Reformen und geldpolitischer Maßnahmen findet sich in Drudi et al. (2012) und in European Central Bank (2012a). Für einen Vergleich der Finanzkrise zu den Erfahrungen in den USA und in Japan während der 1990er Jahre siehe European Central Bank (2012c).

3 Es ist nicht der Anspruch dieses Beitrages, alle Facetten des Neofunktionalismus zu testen/ substantivieren. Wir beschränken uns dabei auf einige Grundannahmen bzw. eine Grunddynamik des Neofunktionalismus (siehe Abschnitt 4). Ausführlichere (integrations-)theoretische Debatten zu diesem EU-Politikfeld sind u.a. zu finden in: Sandholtz (1993); Grieco (1995); Moravcsik (1998: Kap. 6); Risse et al. (1999); Verdun (2007); Schimmelfennig (2012). 
sich Abschnitt 5, um zu erklären, wie solch weitreichende Reformen politische Zustimmung finden konnten und wie die Geschwindigkeit der Reformen in schwierigen Zeiten erreicht werden konnte. In Abschnitt 6 ziehen wir unsere Schlussfolgerungen.

\section{Die Europäische Antwort auf die Krise}

Die Reaktion der Staaten auf den bereits eingangs illustrierten Krisenverlauf bewirkte wichtige Veränderungen auf globaler, regionaler und nationaler Ebene sowohl im öffentlichen Bereich als auch im Finanzsektor. Erste Maßnahmen als Antwort auf die Krise wurden auf globaler Ebene von den Staats- und Regierungschefs vereinbart. Das Forum der G20-Staaten wurde als zentrales, globales Diskussionsund Entscheidungsinstrument gestärkt und der Internationale Währungsfonds etablierte sich als wichtiges Handlungsinstrument. Die Vereinbarungen hatten als Hauptziel, das globale Finanzsystem durch eine striktere Kontrolle des Finanzsektors sowie eine verbesserte internationale Koordination innerhalb der bestehenden Rahmenbedingungen zu stärken. ${ }^{4}$ Die EU als wesentlicher Teil der globalen Wirtschaft entwickelte, verabschiedete und implementierte die internationalen Vereinbarungen. ${ }^{5}$ Die bestehende EU-Gesetzgebung wurde im Einklang mit dem übergeordneten Arbeitsprogramm verstärkt. ${ }^{6}$ Im Gegensatz zu den global-koordinierten Reformen wurde auf europäischer Ebene ein verschärftes Regelwerk durch die Schaffung neuer Institutionen ergänzt. EU-Mitgliedsstaaten reagierten auf die landesspezifischen Herausforderungen u.a., indem neue Behörden etabliert und Regeln verschärft sowie neue Initiativen in die europäischen Entscheidungsgremien eingebracht wurden. ${ }^{7}$

Die europäischen Reformen in der Überwachung auf Makro- und Mikroebene, welche im Fokus dieses Beitrags stehen, müssen im breiteren Kontext anderer weitreichender institutioneller Veränderungen in der Eurozone hinsichtlich der Finanzund Fiskalstabilität und wirtschaftlicher Reformen betrachtet werden. Die wirtschaftspolitische Koordination und zwischenstaatliche Solidarität wurde im Rahmen der Krisenbewältigung mit dem Ziel verstärkt, die Mitgliedstaaten des Euroraums dazu zu befähigen, sich bei Notwendigkeit unter strengen Bedingungen gegenseitig finanziell unterstützen zu können, um die Finanzstabilität der Eurozone zu wahren. Der erste unternommene Schritt in diese Richtung war die Bewilligung der zwar bilateral bereitgestellten, aber trotzdem koordinierten, Kredite für Grie-

4 Siehe die Erklärung der G20 (Department of Finance Canada 2009). Für eine kurze Chronologie der Arbeiten des IWF für die G20 siehe International Monetary Fund (2012).

5 Siehe European Commission (2010a) sowie ferner die Studie zu grenzüberschreitenden Bankangelegenheiten während der Finanzkrise. Vgl. European Commission (2012a).

6 Für eine detailliertere Beschreibung der Reformen zur Regulierung siehe European Commission (2012b).

7 Für einen Überblick der nationalen Maßnahmen siehe European Central Bank (2010). Für einen detaillierten zeitlichen Ablauf der während der Krise auf europäischer Ebene getroffenen Entscheidungen siehe: http://www.ecb.int/ecb/html/crisis.en.html; 10.3.2013. 
chenland: Die sogenannte Kreditfazilität Greek Loan Facility bündelte die finanzielle Unterstützung der Mitgliedstaaten der Eurozone für Griechenland, als Athen im Mai 2010 seinen Zugang zu den internationalen Kapitalmärkten zu vertretbaren Kosten verlor. $\mathrm{Zu}$ diesem Zeitpunkt wurde auch die Entscheidung getroffen, die Europäische Finanzstabilisierungsfazilität (EFSF) zu etablieren, die ein paar Monate später die Form einer vorübergehenden Zweckgesellschaft mit robusterer Infrastruktur und stabileren Verfahren annehmen sollte. Die Aufgaben des EFSF wurden im September 2012 auf den dauerhaften Europäischen Stabilitätsmechanismus (ESM) übertragen, wobei der EFSF für eine Übergangszeit noch für bestehende Hilfsprogramme genutzt wird. Neben diesen in der Eurozone unternommenen Anstrengungen hat die gesamte EU diesen Prozess durch den Europäischen Finanzstabilisierungsmechanismus (EFSM) sowie mithilfe des EU-Haushalts unterstützt. Darüber hinaus wurde die Europäische Investitionsbank durch zusätzliches Kapital gestärkt, unter anderem um Wachstumsimpulse zu geben.

Bezüglich der fiskalischen und wirtschaftlichen Steuerung haben die EU im Allgemeinen und die Eurostaaten im Besonderen den Rahmen für die Haushaltsdisziplin gestärkt (oft als »Stabilitäts- und Wachstumspakt« bezeichnet) und diese Maßnahmen mit strengeren Bestimmungen für nationale finanzpolitische Regeln und einem Verfahren für makroökonomische Ungleichgewichte (VMU) flankiert, um die angemessene Umsetzung der Wirtschaftspolitik im Kontext der WWU sicherzustellen und große wirtschaftliche Ungleichgewichte zu vermeiden. ${ }^{8}$ Die Einhaltung von Haushaltsdisziplin wurde ferner gesichert durch die Annahme des Vertrages über Stabilität, Koordinierung und Steuerung (der sog. »Fiskalpakt «) ${ }^{9}$ sowie durch die von der Kommission vorgeschlagene »Doppel-Verordnung« zur wirtschafts- und haushaltspolitischen Überwachung und Korrektur exzessiver Defizite bei Staaten der Eurozone (European Commission 2011).

All diese Initiativen haben die Koordinations- und Kontrollmechanismen auf EUEbene gestärkt und fielen mit dem Bedarf der Mitgliedstaaten nach gegenseitiger finanzieller Unterstützung zusammen. Die Hauptmotivation bestand in der Errichtung von verbesserten wirtschaftlichen und haushaltspolitischen Steuerungsmöglichkeiten, um gemeinsam mit der Leistung angemessener und an Bedingungen geknüpfter finanzieller Unterstützung mittelfristig zu erreichen, dass fiskalische und makroökonomische Ungleichgewichte nicht länger die Stabilität der WWU bedrohen sollten. ${ }^{10}$

8 Die als »six-pack« bezeichneten Gesetzgebungsmaßnahmen wurden im November 2011 angenommen. Ziel der Maßnahmen ist eine verbesserte haushaltspolitische Überwachung und Koordinierung der Wirtschaftspolitiken der Mitgliedsstaaten und die Vermeidung makroökonomischer Ungleichgewichte im Euro-Währungsgebiet.

9 Der Vertrag über Stabilität, Koordinierung und Steuerung in der Wirtschafts- und Währungsunion (VSKS) wurde als zwischenstaatlicher Vertrag von den Mitgliedstaaten der Eurozone angenommen. Sein Hauptziel ist es, Haushaltsregeln in den nationalen Gesetzesrahmen auf Verfassungs- oder äquivalenter Ebene einzuführen. Dies wird nach den Vorschlägen der Kommission zu Prinzipien für automatische Korrekturmechanismen geschehen, deren Umsetzung in nationales Recht gerade vom EuGH geprüft wird.

10 Detaillierte Angaben zu diesen Reformen sind zu finden in: European Central Bank (2011) und European Commission (2012c). 
Eine vollständige Analyse aller Querverbindungen und Abhängigkeiten der Reforminitiativen auf globaler, EU-, Euroraum- und nationaler Ebene sind für ein umfassendes Verständnis dieser Entwicklungen zwar wünschenswert, jedoch in den Grenzen dieses Artikels nicht zu leisten. Der übrige Teil des Beitrags konzentriert sich daher auf die jüngsten Reformen der Vereinbarungen zur Überwachung auf EU-Ebene und analysiert diese aus einer theoretisch-neofunktionalistischen Perspektive.

\section{Von der Kooperation auf Ausschussebene zu einem einheitlichen Überwachungsmechanismus}

Ausgangspunkt der Reform, die in einem einheitlichen Aufsichtsmechanismus enden soll, war eine relativ junge institutionelle Infrastruktur, die sich in den vergangenen zehn Jahren in einem evolutionären Prozess entwickelt hatte. Während der frühen 2000er Jahre verfolgte die Koordination der Bankenüberwachung auf europäischer Ebene einen evolutionären, ausschussbasierten Ansatz innerhalb des sogenannten Lamfalussy-Verfahrens. ${ }^{11}$ Nach diesem Ansatz besteht die EU-weite Gesetzgebung und deren konsistente Implementierung aus vier »Ebenen«, was zur Schaffung von lediglich mit Beratungsfunktionen versehenen Ausschüssen geführt hatte. Diese waren mit Experten aus den zuständigen nationalen Behörden besetzt. ${ }^{12}$ Diese Struktur wurde jedoch nie als endgültiges Ziel betrachtet, sah der Lamfalussy-Bericht doch bereits eine Überprüfung des Verfahrens durch die sogenannte Interinstitutionelle Beobachtungsgruppe vor, welche mögliche Engpässe identifizieren sollte. ${ }^{13}$ Darüber hinaus unternahm der Europäische Rat mehrere Versuche zur Verbesserung der Funktionsfähigkeit des bestehenden Verfahrens. ${ }^{14}$

Ein wichtiger Schritt zu einem institutionellen Ansatz wurde mit dem bereits beschriebenen ESFS erreicht, der 2011 seine Arbeit aufnahm. Die drei neuen Europä-

11 Das Konzept wurde ursprünglich im März 2001 entwickelt und ist nach seinem Urheber Alexandre Lamfalussy, dem Vorsitzenden des Beratungsausschusses, benannt. Es besteht aus vier »Ebenen«, wobei sich jede mit einer bestimmten Stufe der Umsetzung der Gesetzgebung beschäftigt (European Commission 2012d).

12 Der Level-2-Ausschuss setzte sich aus Ministerialbeamten zusammen; der Level-3-Ausschuss aus nationalen Aufsichtsbehörden. Für weitere Informationen zum LamfalussyVerfahren siehe European Central Bank (2007).

13 Die Gruppe umfasste sechs unabhängige Experten und nahm ihre Arbeit 2005 auf. In ihrem Abschlussbericht 2007 stellte die Gruppe zwar fest, dass Fortschritte erzielt wurden, identifizierte jedoch auch Probleme bei der Durchsetzung und bezüglich der Vereinbarungen zum Krisenmanagement (Inter-Institutional Monitoring Group 2007).

14 So hat der Europäische Rat im Dezember 2007 die Kommission ersucht, die Rolle des Ausschusses der Aufsichtsbehörden eindeutiger festzulegen und alle Optionen zur Stärkung der Funktionsweise dieser Ausschüsse zu prüfen. 2008 forderte der Europäische Rat eine schnelle Verbesserung der Funktionsweise des Ausschusses der Aufsichtsbehörden sowie weiterhin die Kommission auf, ihre Beschlüsse über die Einsetzung der Ausschüsse der Aufsichtsbehörden im Hinblick auf Kohärenz und Schlüssigkeit der Mandate zu überprüfen. Eine Übersicht des Verfahrens und der Entscheidungen findet sich in European Commission (2009: insb. Rezital 4-6). 
ischen Aufsichtsbehörden (ESA) ersetzten die drei Level-3-Ausschüsse des Lamfalussy-Verfahrens und waren mit einem stärkeren institutionellen Status sowie erweiterten Kompetenzen ausgestattet. ${ }^{15}$

Diese Entscheidungen erwiesen sich jedoch als unzureichend zur Überwindung der Krise, vor allem da die Mitglieder der Eurozone Anfang 2012 mit neuen Herausforderungen im Rahmen der Krisenbewältigung konfrontiert wurden. Der bereits beschriebene sovereign-bank nexus trat deutlicher zutage und ließ sich mit dem bestehenden Instrumentarium nicht mehr effizient genug begegnen. Dieser Nexus wurde insbesondere deutlich, als die Eurostaaten im Frühjahr 2012 ihre Absicht zur Anwendung der neu geschaffenen Instrumente des EFSF signalisierten, um die Rekapitalisierung der spanischen Banken zu unterstützen. Allerdings führte diese durch die spanische Regierung angekündigte Unterstützung des spanischen Bankensektors nicht zur beabsichtigten Entlastung der spanischen Wirtschaft. Ganz im Gegenteil entfaltete das geschnürte Paket sogar eine partiell negative Wirkung, da private Investoren befürchteten, der bevorrechtigte Gläubigerstatus des EFSF könnte negative Auswirkungen auf die private Bankenbeteiligung an spanischen Schulden haben. Auf diese Weise wurden die Grenzen der existierenden Werkzeuge des Krisenmanagements in der Eurozone offenbart. Vor dem Hintergrund dieser Erfahrung traf die EU Vorbereitungen für mutigere Schritte, welche über die Krisenbewältigung und die Finanzmarktregulierung hinausgehen.

2012 unternahmen die Staats- und Regierungschefs vor dem Hintergrund des Berichts der vier Präsidenten ${ }^{16}$ und anderer Empfehlungen wie dem »Liikanen-Bericht« (Liikanen et al. 2012) schrittweise Versuche, den offensichtlichen Unzulänglichkeiten im Finanzsektor und seiner Überwachung zu begegnen.

Das Ziel der Reformen in diesem Bereich war die Errichtung eines effizienteren, integrierteren und nachhaltigeren europäischen Überwachungssystems, wobei ein Schwerpunkt auf der Prüfung der Aufgabenverteilung zwischen der nationalen und der europäischen Ebene lag. Anfang 2012 erhielten die vier Präsidenten das Mandat, Vorschläge auszuarbeiten, welche sie schließlich in einem Bericht am 26. Juni 2012 vorlegen und die EU auf eine solidere Grundlage stellen sollten. Im Gegenzug verabschiedete der Europäische Rat vom 28. und 29. Juni 2012 Schlussfolgerungen, die in mancher Hinsicht als bahnbrechend bezeichnet werden können: Darin forderte der Rat die vier Präsidenten zur Fortführung ihrer Arbeit auf und sprach sich gleichzeitig für die zeitnahe Einrichtung eines einheitlichen Überwachungsmechanismus aus. Daraufhin unterbreitete die Kommission am 12. September 2012

15 Die Europäische Bankenaufsichtsbehörde (EBA) ersetzte den Ausschuss der Europäischen Bankenaufsichtsbehörden (CEBS), die Europäische Aufsichtsbehörde für das Versicherungswesen und die betriebliche Altersversorgung (EIOPA) ersetzten den Ausschuss der Europäischen Aufsichtsbehörden für das Versicherungswesen und die betriebliche Altersversorgung (CEIOPS) und die Europäische Wertpapier- und Marktaufsichtsbehörde (ESMA) ersetzte den Ausschuss der Europäischen Wertpapierregulierungsbehörden (CESR).

16 Den Vorsitz der Gruppe der vier Präsidenten hatte der Präsident des Europäischen Rates, der eng mit den Präsidenten der Kommission, der Eurogruppe und der EZB zusammenarbeitete. 
entsprechende Vorschläge. Danach sollten durch diesen Mechanismus im Grunde genommen sämtliche Banken der Eurozone überwacht und dabei an die EZB angegliedert werden (European Commission 2012e; European Commission 2012f).

Die EZB ist als zentrale aufsichtliche Behörde mit weitreichenden Kompetenzen vorgesehen, die im sogenannten einheitlichen aufsichtlichen Mechanismus agieren wird. Mit diesem Mechanismus, dessen rechtliche Grundlage voraussichtlich in der ersten Hälfte 2013 verabschiedet wird, wird die Bankenaufsicht in Europa erstmals in seinen Entscheidungen zentralisiert, was als Paradigmenwechsel bezeichnet werden kann.

\section{Neofunktionalismus und das Konzept des Funktionalen Spillover}

Die globale und die europäische Reaktion auf die Krise fand, wie bereits erwähnt, auf unterschiedlichen Ebenen und oft in parallelen Anstrengungen statt, die den Gesamtzusammenhang und die Dynamiken nicht offensichtlich werden lassen. Der Neofunktionalismus, eine der Haupttheorien regionaler Integration (vgl. Haas 1958; Niemann/Schmitter 2009), trägt dazu bei, eine themenübergreifende Logik und Struktur in diesen individuellen Reformen zu erkennen. Sein Verständnis von Wandel basiert auf der Idee des spillover, dessen Logik sich auch in der europäischen Krisenbewältigung und der institutionellen Reform widerspiegelt.

Die Grundannahmen des Neofunktionalismus können wie folgt zusammengefasst werden: (1) Integration wird als Prozess verstanden. Dieser Idee des Prozesses liegt die Annahme zugrunde, dass sich Integrationsprozesse mit der Zeit fortentwickeln und ihre eigene Dynamik entfalten. (2) Regionale Integration wird außerdem durch eine Vielzahl von unterschiedlichen Akteuren geprägt, wobei diese Akteure zur Koalitionsbildung über Staats- und Verwaltungsgrenzen hinweg fähig sind (Haas 1964: 68-70). (3) Entscheidungen werden von rationalen und ihre Eigeninteressen verfolgenden Akteuren getroffen. Diese sind jedoch dazu in der Lage, aus ihren Erfahrungen beim Treffen gemeinschaftlicher Entscheidungen zu lernen und ihre Präferenzen zu verändern (Haas 1958: 291; Haas 1970: 627). (4) Ein in mehreren Schritten ablaufender Entscheidungsprozess wird gegenüber umfassenden Entwürfen bevorzugt, bei welchen marginal erscheinende Anpassungen oftmals die Folge unerwarteter Konsequenzen vorheriger Entscheidungen sind.

Grundsätzlich werden drei Arten von spillover unterschieden: kultivierter, politischer und funktionaler spillover (Tranholm-Mikkelsen 1991: 4-6). Das Konzept des kultivierten spillover beschäftigt sich mit der Rolle supranationaler Institutionen, welche, einmal geschaffen, ein Eigenleben entwickeln und in der Folge von jenen Akteuren, die sie geschaffen haben, nur schwer zu kontrollieren sind (Pierson 1996: 132-135, 142). Politischer spillover betont die integrative Rolle von Interessengruppen im Integrationsprozess. Der Neofunktionalismus legt den Schwerpunkt auf das Konzept des funktionalen spillover, nach welchem die Entscheidung zur Integration eines bestimmten Bereichs aufgrund funktionaler Sachzwänge nur durch weitere Integrationsschritte erfolgreich umgesetzt werden kann (Lindberg 1963: 10). Da 
wir in der empirischen Analyse in Abschnitt 5 nur die Logik des funktionalen spillover nutzbar machen und plausibilisieren, beschränken wir uns hier auf eine genauere Spezifizierung dieser spillover-Dynamik.

Funktionale spillover-Effekte entstehen aus Drucksituationen, die stets eine Tendenz zur Ausweitung der Integration beinhalten, da intentionale Akteure dazu neigen, ebenso von den funktionalen Notwendigkeiten und Widersprüchen wie auch von den aus diesen entstehenden Kosten und Nutzen geleitet bzw. überzeugt zu werden. Dieser Effekt geht auf die Unfähigkeit eines Großteils der politischen Akteure zurück, langfristiges und zweckorientiertes Verhalten an den Tag zu legen. Vielmehr »stolpern« sie von einer Entscheidung zur nächsten, insbesondere dann, wenn sie sich mit oft unüberschaubaren Aufgaben der regionalen Integration konfrontiert sehen. In diesem Bereich werden Entscheidungen gewöhnlich mit unvollständigen Informationen über ihre Konsequenzen und unter erheblichem Zeitdruck getroffen (Haas 1970: 627). Haas folgt der Annahme mancher Ökonomen wie beispielsweise Pierre Uri, dem Chefökonomen der EGKS in den 1950ern, dass entstehende funktionale und gegenseitige Abhängigkeiten zwischen verschiedenen Produktionssektoren bzw. Politikfeldern unweigerlich zur Vertiefung der Integration führen, da oftmals die Ziele in einem Politikfeld nur dann erreicht werden können, wenn integrative Schritte in anderen Sektoren vollzogen werden (Haas 1958: 372-373; Lindberg 1963:10-11).

In der weiteren wissenschaftlichen Auseinandersetzung mit diesem Konzept wurde festgestellt, dass die Kraft funktionaler spillover-Logiken nicht nur vom Ausmaß der Interdependenz der jeweiligen Politikbereiche abhängt (Niemann 2006: 31). Zwei Aspekte, die wir in Abschnitt 5 wieder aufnehmen werden, beeinflussen, wann und wie stark funktionale Spannungen auf Akteure einwirken. (1) Wenn funktionale Dissonanzen - d.h. Zustände, die eigentlich einen funktionalen spillover-Prozess in Gang setzen sollten - nicht durch weitere Integrationsschritte behoben werden, kann dies Schocks oder Krisen begünstigen, die dann im Prozess ihrer Bewältigung potenzierten funktionalen Druck nach sich ziehen, und letztendlich die notwendigen Integrationsschritte auslösen. (2) Funktionale Strukturen determinieren nicht das Verhalten von Akteuren in einer mechanischen oder vorhersehbaren Weise. Akteure müssen funktionale Logiken auch als plausibel oder zwingend erachten, damit diese auch eine Wirkung entfalten können. ${ }^{17}$ Mit anderen Worten: Funktionaler Druck ist nur so stark, wie er von (wichtigen) Akteuren wahrgenommen beziehungsweise empfunden wird. So hat es in der (europäischen) Integrationsgeschichte immer wieder Fälle gegeben, in denen relativ schwache funktionale Interdependenzen als veritable »Sachzwänge« begriffen wurden, während sehr viel plausiblere funktionale Zusammenhänge (zunächst) ohne integrative Folgen blieben (vgl. Huysmans 2000). Die Überzeugungskraft bzw. der Einfluss funktionaler Logiken auf Entscheidungsträger lässt sich gut an der Entwicklung des politischen Diskurses ablesen. Wenn funktionale spillover-Argumente unter Entscheidungsträ-

17 Dies geht auf Niemanns konstruktivistische/strukturationstheoretische Überarbeitung des Neofunktionalismus zurück (siehe Niemann 2006: 31). 
gern Verbreitung finden, beziehungsweise vielleicht sogar Teil des dominanten politischen Diskurses werden, dann schlagen sie sich auch in politischen Entscheidungen nieder (Niemann 2006: Kapitel 4). Durch derartige Erweiterungen/Erläuterungen des funktionalen spillover-Konzeptes lässt sich auch genauer spezifizieren, wann und wie solche Logiken auf den Politikprozess einwirken.

Wir möchten mit unserer Analyse der Schritte in Richtung einer stärkeren Finanzmarktintegration durch Reformen in den Bereichen der Bankenregulierung und -aufsicht zeigen, dass sich mit dem Konzept des funktionalen spillover (besonders in seiner erweiterten Form) sowohl die jüngsten als auch zukünftige Reforminitiativen dieser Art auf EU-Ebene nachvollziehbar darstellen und erklären lassen.

\section{Funktionaler spillover als Ursache für einen einheitlichen Überwachungsmechanismus}

Das Konzept des funktionalen spillover trägt zu einem besseren Verständnis des EU-Krisenmanagements bei, wenn gezeigt wird, dass aufgrund funktionaler Interdependenzen zwischen Politikbereichen ein ursprüngliches Ziel nur durch weitere integrative Maßnahmen sichergestellt werden kann. Damit derartige funktionale Dissonanzen auftreten können, bedarf es zunächst eines bedeutenden und/oder dringenden ursprünglichen Politikziels. Im Hinblick auf den vorliegenden Fall gab es mindestens ein sehr wichtiges ursprüngliches Ziel: Die erfolgreiche Umsetzung einer gemeinsamen Währung, welche sich im Laufe der Krise hin zu einer Frage der Sicherung der Stabilität der WWU entwickelt hat. Diese wurde mit dem noch grundlegenderen Ursprungsziel verknüpft, den Binnenmarkt zu bewahren. ${ }^{18}$ Sowohl die WWU als auch der Binnenmarkt stellen grundlegende Politikziele dar, die oftmals auch als entscheidend für das gesamte EU-Projekt erachtet werden. ${ }^{19}$

In einem nächsten Schritt widmen wir uns der Ebene der funktionalen Interdependenz (zwischen relevanten Politikfeldern), die in Richtung weiterer notwendiger Integration deutet. Besonders aus neofunktionalistischer Perspektive stellen die institutionellen Entwicklungen in den Eurostaaten und dort insbesondere im Bereich der Finanzaufsicht notwendige Schritte auf dem Weg zur Vollendung der in Maastricht entstandenen Finanz- und Wirtschaftsarchitektur dar. Die Richtung dieser Entwicklung hin zu verstärkter Integration lassen sich über funktionale Dissonanzen erklären, die aus einem unvollständigen und unzureichenden institutionellen Rahmen entstehen.

Die Einführung des Euro und einer einheitlichen Währungspolitik haben die gegenseitigen Abhängigkeiten zwischen den Eurostaaten erheblich verstärkt - insbesondere beim Kapitalmarkt, den Staats- und Firmenanleihen, beim Aktienmarkt

18 Für die (funktionalen) Notwendigkeiten und Verbindungen zwischen dem Binnenmarkt und der Wirtschafts- und Währungsunion (WWU), siehe z.B. Padoa-Schioppa (2000: 83-92).

19 So z.B. Kanzlerin Merkel als sie in der Bundestagsrede vom 27. Februar 2012 erklärte: »Europa scheitert, wenn der Euro scheitert« (Merkel 2012a). 
und im Bankensektor. ${ }^{20}$ Während jedoch im Bankensektor grenzübergreifende Körperschaften und Aktivitäten nach der Euro-Einführung florierten, blieben die allgemeinen politischen Kompetenzen und Weichenstellungen auf die nationale Ebene begrenzt und es wurden keine (ausreichenden) institutionellen Anpassungen vorgenommen, um die Auswirkungen der weit integrierten Währungsunion zu flankieren beziehungsweise zu kompensieren. Besonders die Anreizsysteme in der Bankenaufsicht blieben aufgrund der national ausgerichteten Überwachungssysteme unausgewogen. In Zeiten einer stabilen und/oder wachsenden Wirtschaft war dies zwar nicht notwendigerweise ein Problem. Es erwies sich jedoch als erheblicher Nachteil für das Finanzsystem, wenn nationale Behörden aufgrund krisenbedingter finanzieller Instabilität eingreifen mussten und damit selbst in den Sog der Krise gerieten.

\subsection{Die Krise: Folge bestehender und Verstärker nachfolgender funktionaler Dissonanzen}

Wie bereits in Abschnitt 4 angedeutet: Wenn funktionale Dissonanzen nicht durch weitere Integrationsschritte behoben werden, kann dies Krisen begünstigen, die dann im Prozess ihrer Bewältigung potenzierten funktionalen Druck nach sich ziehen, und letztendlich die notwendigen Integrationsschritte auslösen. Diesen Prozess können wir in der Eurozone (besonders der zweiten Hälfte) der 2000er Jahre beobachten. Nach Einführung der Gemeinschaftswährung, weitgehend auf Basis der Architektur von Maastricht, entwickelten sich zwar funktionale Diskrepanzen zwischen supranationaler Währungspolitik und integrierten Finanzmärkten auf der einen Seite, und intergouvernementaler Fiskalpolitik und nationalen Aussichtsbehörden auf der anderen Seite. Die positive wirtschaftliche Wachstumsentwicklung in den frühen Jahren der Währungsunion hatte jedoch keinen ausreichenden Druck entstehen lassen, um einen funktionalen spillover-Prozess mit ausreichender Geschwindigkeit in Gang zu setzen. ${ }^{21}$ Mit dieser Dissonanz wurden tendenziell Schocks oder Krisen begünstigt, die dann im Prozess ihrer Bewältigung potenzier-

$20 \mathrm{Zu}$ den Entwicklungen in diesen Märkten und zum Verlauf der Integration seit der Einführung des Euro siehe die »Financial Stability Reviews« der EZB seit 2004, die jährlichen »Financial Integration In Europe«-Berichte ebenso wie die EZB-Berichte unter der Bezeichnung »Indicators of Financial Integration in the Euro Area« vom September 2005 (European Central Bank 2005) und 2006 (European Central Bank 2006), abrufbar auf der Homepage der EZB.

21 Die Reform der Aufsichtsstrukturen erfolgte in einem evolutionären Prozess, wie im Abschnitt 3 kurz ausgeführt wurde. Die EZB hat eine schneller und weiter voranschreitende Harmonisierung und Integration der Aufsicht stets angemahnt. European Central Bank (2003) und European Central Bank (2010) analysieren die Vielfalt institutioneller Verantwortlichkeiten auf nationaler Ebene. So heißt es in der Schlussfolgerung zum Lamfalussy Prozess: »Market developments underscore the urgency of enhancing cross-border convergence and cooperation in the supervision of EU banks in order to ensure an effective monitoring of and response to cross-border financial risks and to provide a streamlined supervisory interface and a level playing field for market participants« (ECB 2007: 13). 
ten funktionalen Druck nach sich zogen und letztendlich die notwendigen Integrationsschritte auslösten.

Aus finanzieller Perspektive entstanden durch den Euro zusätzliche Inkonsistenzen, die entsprechend weiteren Druck zur fortschreitenden Integration aufbauten. Schoenmaker (2012) prägte den Begriff des finanziellen »Trilemmas«, und formuliert wie folgt:

\begin{abstract}
»The Freixas-model of cross-border externalities provides the theoretical foundation for the financial trilemma (Schoenmaker 2011). The trilemma is that the three policy objectives - maintaining global financial stability, fostering cross-border financial integration, and preserving national authority - do not easily fit together. [...] Any two of the three objectives can be combined with relative ease, but it is difficult to achieve all three. The financial trilemma forces policymakers to make a choice. Maximising global welfare means considering global financial stability and other global objectives, such as reliability of financial contracting and efficiency of global allocation of funds. As crossborder financial integration progresses, policy makers will have less scope for independent policy-making, including fiscal independence. Ultimately, the trilemma boils down to the issue of sovereignty. At one extreme, policy makers can hand over part of their sovereignty to foster global banking and global financial stability. At the other extreme, policy-makers can choose to impose restrictions on cross-border banking to preserve their full sovereignty (see Cerutti et al. 2010) on the costs of ring fencing). The extensive international reach of European banks suggests that resolving the trilemma is most urgent for Europe« (Schoenmaker 2012: 5).
\end{abstract}

Die Krise hat dann die nüchterne Wahrheit dieses Trilemmas aufgedeckt und die Schwächen der national ausgerichteten Überwachung innerhalb der EU offengelegt, deren evolutionärer Ansatz in scharfem Kontrast zu der sich rapide weiterentwickelnden Krise und insbesondere den Verwicklungen von negativen fiskalischen und finanziellen Entwicklungen stand, welche zu einer problematischen gegenseitigen Abhängigkeit der Banken (dem bereits beschriebenen sovereign-bank nexus) voneinander führten (siehe Abschnitte 2 und 3). Dies führte dazu, dass die einheitliche Währungspolitik nicht im gesamten Euroraum umgesetzt und damit die notwendige Finanzierung der Wirtschaft durch das Bankensystem nicht erreicht werden konnte. Das hieraus resultierende Ergebnis wurde im November 2012 von EZB-Präsident Mario Draghi herausgestellt:

»The crisis has highlighted a fundamental inconsistency between the single monetary policy of the euro area and the responsibility of national authorities for financial policies. The single currency needs a single financial system that is not fragmented along national lines« (Draghi 2012).

Die funktionalen Dissonanzen repräsentierten (also) noch keine zwingende Ursache für einen funktionalen spillover, sondern bauten erst zusammen mit den finanziellen und ökonomischen Zwängen im Rahmen der Finanzkrise einen ausreichenden Druck auf die politischen Entscheidungsträger auf, welcher die Reformen (siehe Abschnitt 3) erst in diesem Umfang und in dieser Geschwindigkeit ermöglichte. Neofunktionalistisch ausgedrückt bündelte die Krise funktionale spillover und fungierte als Katalysator, der die Motivlagen der politischen Entscheidungsträger dahingehend anpasste, dass sie sich in Richtung einer weiteren Vergemeinschaftung der Strukturen auf europäischer Ebene bewegten. Dieser Fortschritt auf 
dem Weg zur Konsensfindung unter den Mitgliedstaaten war weniger ein Selbstzweck, sondern eher der Notwendigkeit geschuldet, das Ziel eines gut funktionierenden Steuerungsrahmens und die Finanzstabilität auf europäischer Ebene zu erreichen.

Der funktionale spillover fand dabei in allen drei der WWU zugehörigen Bereichen statt, also im wirtschaftlichen, fiskalischen und finanziellen. Dies spiegelt zu einem gewissen Grad die Querverbindungen der Märkte untereinander wider. Öffentliche und private Überschuldung sowie makroökonomische Ungleichgewichte bedeuteten, dass die fiskalischen und wirtschaftlichen Rahmenbedingungen durch institutionelle Verbesserungen wie dem six-pack und dem Fiskalpakt (siehe Abschnitt 2) strenger gefasst werden mussten. Der Zugang zu finanzieller Unterstützung durch den EFSF und den ESM wurde an die Übernahme und Folgeleistung der neuen fiskalischen und wirtschaftlichen Rahmenbedingungen geknüpft. Tatsächlich kann der Fiskalpakt als der »Einsparungsteil« und der ESM als der »Solidaritätsteil« eines zweigleisigen Ansatzes zur Vollendung der wirtschaftlichen Rahmenbedingungen mitten in der Krise verstanden werden.

Der Aufsichtsmechanismus wurde als Teil der Gesamtreform mit dem ESFS und dem zukünftigen Single Supervisory Mechanism (SSM) weiterentwickelt und kann als Segment des »Kontrollteils«, und damit als dritte Dimension verstanden werden. ${ }^{22}$ Dieser Teil rückte vor allem in den Fokus, da der ESM durch die neu geschaffene Möglichkeit der direkten Bankenrekapitalisierung dem sogenannten Risiko des moral hazard ${ }^{23}$ Vorschub leisten könnte, falls Möglichkeiten der Überwachung allein auf der nationalen Ebene verbleiben. Aus diesen Gründen wurde eine Überwachungsvereinbarung jenseits nationaler Mandate notwendig. ${ }^{24}$ Weitere parallele Entwicklungen im Aufsichtsbereich haben sich später als Fehler herausgestellt. Dazu gehören etwa die zwei Stresstests der EBA, die befriedigende Ergebnisse bezüglich der Risiken für die Finanzstabilität in der EU und der Eurozone erreichen sollten. Sie scheinen zumindest teilweise Folge des bereits erwähnten Trilemmas gewesen zu sein und damit die Notwendigkeit einer supranationalen Finanzaufsicht verstärkt zu haben. Die Etablierung des SSM und die Reform der EBA bilden in diesem Sinne eine sinnvolle Entwicklung hin zu einer weitergehenden Integration.

Eine Reform der europäischen Bankenaufsicht bleibt jedoch problematisch, solange sie nicht in einen konsistenten Kontext eingebettet ist. Insbesondere die Finanzierung einer Bankenrekapitalisierung oder der Einlagensicherung stellt eine Dimension des bank-sovereign nexus dar, die weiteren Anpassungsdruck ausübt.

22 Er hatte jedoch selbst wiederum weitere technische Auswirkungen auf die bestehende EU-Rechtsetzung. Dies passierte durch die sog. Omnibus-Richtlinie (Directive/2010/78/ EU).

23 Moral hazard bezieht sich auf die Vorstellung, dass Marktteilnehmer (z.B. Banken) exzessive Risiken mit dem Wissen eingehen, dass sie im Falle des Falles ohnehin gerettet werden würden (bspw. durch den ESM).

24 Ein weiterer Grund für ihre Notwendigkeit bestand darin, unabhängiger von den nationalen Regierungen operieren zu können und eine robuste Anreizstruktur und Unabhängigkeit bei den Überwachungsentscheidungen zu gewährleisten. 
Vor dem Hintergrund der multidimensionalen Natur der Krise erforderte eine tragfähige Antwort einen weiter gefassten Ansatz. Die vier Präsidenten trugen dieser Notwendigkeit Rechnung und erarbeiteten eine umfassendere Vorstellung des europäischen Wegs. Der bedeutendste Vorschlag der vier Präsidenten zur Finanzmarktunion, einem der vier bereits erwähnten Grundbausteine, war die Neuverteilung der Bankenaufsicht in den Staaten der Eurozone (und anderer hieran interessierter EU-Staaten) zu einem gemeinsamen Überwachungsmechanismus unter Beteiligung der EZB (Van Rompuy 2012a). Hinsichtlich der Bankenaufsicht kam die Analyse der vier Präsidenten zu dem Schluss, dass ein gemeinsamer Überwachungsmechanismus auf europäischer Ebene jedoch auf lange Sicht die Finanzstabilität nicht alleine gewährleisten kann, ohne durch weitere Instrumente und Institutionen flankiert zu werden. Diese Instrumente bestanden hauptsächlich aus einem einheitlichen Rechtsrahmen, dem sogenannten »Integrierten Finanzrahmen«, und weiteren Krisenbewältigungsmechanismen. Dazu gehören eine Behörde und ein Fonds zur Krisenbewältigung für Banken sowie ein gemeinsames Einlagensicherungssystem (Van Rompuy 2012b).

\subsection{Zunehmende Prägung des politischen Diskurses durch funktionale Logik}

Wie in Abschnitt 4 beschrieben, determinieren funktionale Strukturen das Verhalten von Akteuren nicht in vorhersehbarer Weise. Akteure müssen funktionale Logiken auch als plausibel oder zwingend erachten, damit diese auch eine Wirkung entfalten können. Die Krise als Folge bestehender, und Verstärker nachfolgender funktionaler Dissonanzen hat offenbar Lehreffekte forciert und die Logik des funktionalen spillover noch einmal entscheidend erhöht. Dies spiegelt sich auch im politischen Diskurs auf europäischer und nationalstaatlicher Ebene wider. Obwohl die funktionale spillover-Dynamik, die von der WWU ausgeht, schon seit spätestens Ende der 1980er Jahre (eher vereinzelt) artikuliert wurde, ${ }^{25}$ so hat sie lange Zeit keine entscheidende Rolle im politischen Diskurs gespielt.

Mit der Krise scheint die funktionale spillover-Argumentation relativ schnell zum dominanten politischen Diskurs geworden zu sein. Dieser scheint nahezu alle nationalen Regierungen und EU-Institutionen zu durchziehen. Dies fängt an bei der funktionalen Verknüpfung zwischen dem Binnenmarkt und der gemeinsamen Währung, die inzwischen von einer Vielzahl von Politikern angeführt wird. Nach Bundesfinanzminister Wolfgang Schäuble zum Beispiel »besteht durchaus die Gefahr, dass bei einem Auseinanderbrechen des Euro [...] vieles von dem, was wir erreicht und liebgewonnen haben, in Frage gestellt würde - vom gemeinsamen Binnenmarkt bis hin zur Reisefreiheit in Europa« (Der Spiegel 2012a). Ähnlich argumentiert der spanische Premierminister Rajoy, der in einem Brief an den Kommissionspräsidenten José Manuel Barroso und den Präsidenten des Europäischen Rates Herman Van Rompuy behauptet: »The euro is on a path of no return, and its connec-

25 Siehe Delors Committee (1989); Deutsche Bundesbank (1990). 
tion with the entire European project, starting with the common market, is indissolvable« (Rajoy 2012).

Auch was die funktionalen Erfordernisse und Konsequenzen der gemeinsamen Währung anbelangt, bedienen sich Politiker in Europa vermehrt der neofunktionalistischen spillover-Logik. In den Worten von Bundeskanzlerin Angela Merkel ausgedrückt: »Eine erneuerte Wirtschafts- und Währungsunion braucht mehr gemeinsame Fiskalpolitik, zudem eine gemeinsame Wirtschaftspolitik« (Der Spiegel 2012b). Dabei scheut sich die Kanzlerin nicht, den Delors-Bericht von 1989 zu zitieren, der bereits damals von einer notwendigen weitreichenden wirtschaftlichen Integration als Folge einer Währungsunion gesprochen hatte (Delors Committee 1989), um dann zu konstatieren: »Die europäische Staatsschuldenkrise um den Euro zeigt, wie scharfsichtig und richtig die Analyse Jacques Delors' war« (Merkel 2012b). Mit den Worten des derzeitigen Kommissionspräsidenten Barroso klingt die funktionale Verbindung wie folgt: »It was an illusion to think that we could have a common currency and a single market with national approaches to economic and budgetary policy« (Barroso 2011: 4). Ähnlich, aber umfassender, formulieren es die vier Präsidenten:

»The actions deemed necessary to ensure the resilience of the EMU are presented therein as a staged process. Irrespective of their time horizon, all policy proposals have been conceived and designed as elements of a path towards a genuine Economic and Monetary Union. Given the strong linkages between the building blocks, they should be examined as part of a mutually reinforcing comprehensive package. The creation of an integrated financial framework has important fiscal and economic implications and therefore cannot be envisaged separately. Similarly, the proposals put forward in the fiscal and economic policy sphere are closely intertwined. And, as all the proposals imply deeper integration, democratic legitimacy and accountability are essential to a genuine Economic and Monetary Union« (Van Rompuy et al. 2012: 3).

Den eng mit dieser Thematik verbundenen funktionalen Brückenschlag zwischen der WWU und einer nicht immer ganz klar definierten politischen Union macht inzwischen auch eine Vielzahl von Entscheidungsträgern auf nationaler und europäischer Ebene. ${ }^{26}$ Neben Bundeskanzlerin Merkel und den vier Präsidenten folgt unter anderem auch der französische Präsident François Hollande dieser Logik: »This eurozone must take a political dimension [... but] political union comes afterwards. It is the step that follows the fiscal union, the banking union, the social union« (Le Monde 2012). In weiten Kreisen des Europäischen Parlaments und der Europäischen Kommission scheint eine politische Union als notwendige Folge der ge-

26 Auch in Vertragstexten ist die funktionale spillover-Logik ausgehend von der WWU erkennbar. Im Vertrag über Stabilität, Koordinierung und Steuerung der Wirtschafts- und Währungsunion, der im März 2012 von 25 Staats- und Regierungschefs unterzeichnet wurde, wird darauf verwiesen, dass die Vertragsparteien für das »reibungslose Funktionieren der Wirtschafts- und Währungsunion gemeinsam auf eine Wirtschaftspolitik hinarbeiten müssen, bei der sie gestützt auf die in den Verträgen, auf denen die Europäische Union beruht, festgelegten Mechanismen der wirtschaftspolitischen Koordinierung in allen für das reibungslose Funktionieren des Euro-Währungsgebiets wesentlichen Bereichen die notwendigen Schritte und Maßnahmen einleiten [...]« (European Council 2012b). 
meinsamen Währung, besonders im Zuge der Krise, wenig kontrovers. So formuliert z.B. EU-Kommissar Joaquín Almunia:

»Es ist legitim, nationale Interessen zu verfolgen. Aber am Ende müssen alle Länder das machen, was Europa als Ganzes rettet. Deshalb haben die Deutschen recht, wenn sie nach der Wirtschafts- und Währungsunion auch eine politische Union fordern. Da stimme ich voll mit ihnen überein« (Spiegel Online 2012b).

Schritte in Richtung einer europäischen Finanzmarkt- und Bankenunion, die in diesem Beitrag besondere Aufmerksamkeit erfahren, werden im politischen Diskurs auch größtenteils als logische Folge funktionaler Sachzwänge konstruiert. Dies tun sogar Politiker euroskeptischerer Staaten, die vielleicht eher bemüht sein sollten, die Grenzen nationaler Souveränität zu unterstreichen. Der britische Finanzminister George Osborne etwa lässt verlauten: »We've always said a banking union was a necessary part of a more stable single currency for the Eurozone [...] (The Guardian 2012). Noch ausführlicher formulieren dies die vier Präsidenten:

»Den derzeitigen europäischen Vorkehrungen für die Wahrung der Finanzstabilität fußen nach wie vor auf nationalen Zuständigkeiten. Dies ist mit dem hochintegrierten Charakter der WWU nicht zu vereinbaren und hat das schädliche Wechselspiel zwischen der Anfälligkeit der Staaten und den Schwachstellen des Bankensektors noch verschärft. Mit dem einheitlichen Aufsichtsmechanismus wird ein Garant für eine strenge und unparteiliche Aufsicht geschaffen, was zur Auflösung der Verbindung zwischen Staaten und Banken beitragen und wird die Wahrscheinlichkeit künftiger systemischer Bankenkrisen verringern wird« (Van Rompuy et al. 2012: 5).

Die vorangegangene Illustrierung weist darauf hin, dass eine zunehmende Akzeptanz funktionaler Sachzwänge unter wichtigen Entscheidungsträgern nach der Krise den politischen Diskurs dominiert. Dies scheint zu zeigen, dass der funktionale Druck im Laufe der Jahre und mit Entwicklung der Krise auch (zunehmend) als solcher durch die politischen Eliten Europas wahrgenommen wurde. Da Diskurse in der Regel Handlungsoptionen von Entscheidungsträgern im politischen Prozess einschränken (Jachtenfuchs 1997: 47), lässt sich tentativ folgern, dass dieser Diskurs sich auch in den politischen Entscheidungen der handelnden Politiker manifestierte.

\section{Fazit}

Während der Finanzkrise ist die Europäische Integration in einem (sehr) schnellen Tempo vorangeschritten. Ausgangspunkt dieser Entwicklung ist die mit dem Vertrag von Maastricht errichtete Wirtschafts- und Währungsunion, welche den freien Personen-, Güter-, Kapital- und Dienstleistungsverkehr zwischen den Mitgliedstaaten mit einer einheitlichen Währung der Eurostaaten mit nationaler Unabhängigkeit in der Finanzpolitik verband. Das europäische Finanzsystem zeigte in einigen Bereichen eine fortschreitende Integration, blieb aber in vielen Bereichen fragmentiert und auch dessen Aufsicht blieb eine nationale Aufgabe auf Basis von harmonisierten Regeln und Standards. Die volle Größenordnung dieses Problems offenbarte sich in der Krise, als die nationalen Staatsschulden und die nationalen Bankensyste- 
me sich in einem »Teufelskreis« gegenseitiger Abhängigkeiten und Ansteckungen wiederfanden. Europa stand vor der Wahl, eine Renationalisierung der Politikbereiche zu akzeptieren oder die europäische Integration entschieden voranzutreiben. Die Entscheidung für Europa und das Festhalten an der europäischen Idee sind von den politischen Entscheidungsträgern oft unter Beweis gestellt worden - wurden wiederholt als alternativlos bezeichnet - und bildeten den Anker und Kompass für die gegenwärtigen Reformen.

Dieser Beitrag zeigt, wie sich diese Entwicklung mithilfe des funktionalen spillover-Konzeptes erklären lässt. Zunächst wurde festgestellt, dass im Kontext der WWU einige wichtige ursprüngliche Politikziele vorliegen (wie z.B. die Bewahrung des Binnenmarktes oder die Stabilisierung der WWU), deren Realisierung weitere integrative Schritte erforderlich macht aufgrund der funktionalen Interdependenz verschiedener involvierter Politikfelder. Die Einführung einer einheitlichen Währungspolitik hat zu einigen funktionalen Dissonanzen geführt: Während z.B. im Bankensektor grenzübergreifende Körperschaften und Aktivitäten nach der Euro-Einführung florierten, blieben die allgemeinen politischen Kompetenzen und Weichenstellungen auf die nationale Ebene begrenzt und es wurden keine adäquaten institutionellen Anpassungen vorgenommen, um die Auswirkungen der weit integrierten Währungsunion zu flankieren. Während dies in Zeiten einer stabilen Wirtschaft kein größeres Problem darstellte, erwies es sich allerdings als erheblicher Nachteil für das Finanzsystem in Zeiten krisenbedingter finanzieller Instabilität. So zog die Krise, die selbst durch funktionale Dissonanzen einer nur währungspolitisch ausreichend integrierten WWU begünstigt wurde, im Prozess ihrer Bewältigung potenzierten funktionalen Druck nach sich. Die Krise bündelte und verstärkte funktionale Sachzwänge, wodurch sich die Motivlagen der politischen Entscheidungsträger dahingehend anpassten, dass sie sich in Richtung einer weiteren Vergemeinschaftung der Strukturen auf europäischer Ebene bewegten. Die funktionale spillover-Logik spiegelt sich auch im politischen Diskurs seit Ausbruch der Krise wider. Entscheidungsträger auf nationaler und europäischer Ebene rekurrieren vielfach auf diese Logik, um die Maßnahmen des Krisenmanagements zu begründen.

Man könnte die These wagen, dass die Vision einer WWU mit vier genuinen fiskalischen, wirtschaftlichen, finanziellen und politischen Unionen (wie sie von den vier Präsidenten sowie gesondert von der Europäischen Kommission umrissen wurde und die der Europäische Rat in Teilen bereits aufgenommen hat) an sich bereits nahelegt, dass das Gerüst der WWU weiter ausgebaut werden muss, um den funktionalen spillover-Druck zu reduzieren. Im Rahmen der Finanzmarktunion werden folglich einige Elemente wie eine europäische Abwicklungsbehörde für nicht-überlebensfähige Banken als selbstverständliche Ergänzung zum einheitlichen Aufsichtsmechanismus vorgesehen. Diese Komplementarität gilt, auch wenn diese Institutionen nicht zeitgleich aufgebaut werden. Konkret besteht im Frühjahr 2013 die Abwicklungsbehörde nur als Konzept, während sich der einheitliche Aufsichtsmechanismus bereits im Anfangsstadium eines Aufbaus befindet. Darüber hinaus sind noch weitere mögliche Szenarien vorstellbar. Beispielsweise kann auf Basis des ge- 
nannten Berichts vermutet werden, dass diese Schritte im Rahmen der Finanzmarktunion mit einer weiteren Vertiefung der fiskalischen Integration in Europa verknüpft sind, welche sowohl ein Mehr an nachhaltigen politischen Initiativen und durchsetzbaren Regeln als auch an fiskalischer Leistungskraft auf europäischer Ebene umfassen.

Während sich schwer voraussagen lässt, wie genau sich diese Vision und ihre Funktionszusammenhänge letztlich entwickeln werden, erscheinen sie zumindest eine Anerkennung des verbleibenden und zu erwartenden funktionalen Drucks zu sein. Sofern diese als Weitsicht europäischer Politiker verstanden werden kann, in dem Sinne, dass Entscheidungsträger funktionale spillover-Effekte antizipieren und ihnen zuvorkommen können, handelt es sich dabei möglicherweise um ein Novum in der Geschichte des europäischen Integrationsprozesses und könnte sich als ein positives und ermutigendes Zeichen für seine zukünftige Entwicklung erweisen.

\section{Literatur}

Barroso, José M. 2011: European Renewal - State of the Union Address 2011 (Europäisches Parlament, Straßburg, 28.9.2011), in: http://europa.eu/rapid/press-release_SPEECH-11607_en.htm; 9.3.2013.

Delors Committee 1989: Report on Economic and Monetary Union in the European Community (Delors Report, Committee for the Study of Economic and Monetary Union, EC Publications Office), Luxemburg.

Department of Finance Canada 2009: Declaration on Further Steps to Strengthen the Financial System, 5.9.2009, London, in: http://www.g20.utoronto.ca/2009/2009banking0905.h tml; 9.3.2013.

Der Spiegel 2012a: Finanzminister Schäuble über die Geburtsfehler der Gemeinschaftswährung und den Abschied vom Nationalstaat, in: http://www.spiegel.de/spiegel/finanzminister-schaeuble-ueber-die-geburtsfehler-des-euro-a-840867.html; 9.3.2013.

Der Spiegel 2012b: Europa - Die Kuhhändler, in: http://www.spiegel.de/spiegel/print/ d-90254946.html; 9.3.2013.

Deutsche Bundesbank 1990: Statement by the Deutsche Bundesbank on the Establishment of an Economic and Monetary Union in Europe: Monthly Report of the Deutsche Bundesbank, October 1990, 40-44.

Directive 2010/78/EU of the European Parliament and of the Council of 24 November 2010 $[\ldots]$ in respect of the powers of the European Supervisory Authority (European Banking Authority), the European Supervisory Authority (European Insurance and Occupational Pensions Authority) and the European Supervisory Authority (European Securities and Markets Authority), in: http://eur-lex.europa.eu/LexUriServ/LexUriServ.do?uri=OJ:L: 2010:331:0120:0161:EN:PDF; 9.3.2013.

Draghi, Mario 2012: Rationale and Principles for Financial Union (Speech by Mario Draghi, President of the ECB, at the 22nd Frankfurt European Banking Congress, Frankfurt am Main, 23.11.2012), in: http://www.ecb.int/press/key/date/2012/html/sp121123.en.html; 10.3.2013.

Drudi, Francesco/Durré, Alain/Mongelli, Francesco 2012: The Interplay of Economic Reforms and Monetary Policy: The Case of the Eurozone, in: Journal of Common Market Studies 50: 6, 881-898.

European Central Bank 2003: Developments in National Supervisory Structures, June 2003, in: http://www.ecb.europa.eu/pub/pdf/other/supervisorystructureen.pdf; 9.3.2013. 
European Central Bank 2005: Indicators of Financial Integration in the Euro Area: September 2005, in: http://www.ecb.int/pub/pdf/other/indicatorsfinancialintegration200509en.pdf; 9.3.2013.

European Central Bank 2006: Indicators of Financial Integration in the Euro Area: September 2006, in: http://www.ecb.int/pub/pdf/other/indicatorsfinancialintegration200609en.pdf; 9.3.2013.

European Central Bank 2007: Review of the Lamfalussy Framework Eurosystem Contribution: November 2007, in: http://www.ecb.europa.eu/pub/pdf/other/lamfalussy-review2007en.pdf; 9.3.2013.

European Central Bank 2010: Measures Taken by Euro Area Governments in Support of the Financial Sector: Monthly Bulletin April 2010, in: http://www.ecb.europa.eu/pub/pdf/ other/art2_mb201004en_pp75-90en.pdf; 9.3.2013.

European Central Bank 2011: Monthly Bulletin March 2011, in: http://www.ecb.int/pub/pdf/ $\mathrm{mobu} / \mathrm{mb} 201103 \mathrm{en} . \mathrm{pdf} ;$ 9.3.2013.

European Central Bank 2012a: Key Dates of the Financial Crisis (since December 2005), in: http://www.ecb.int/ecb/html/crisis.en.html; 9.3.2013.

European Central Bank 2012b: Opinion of the European Central Bank of 27 November 2012 on a Proposal for a Council Regulation Conferring Specific Tasks on the European Central Bank Concerning Policies Relating to the Prudential Supervision of Credit Institutions and a Proposal for a Regulation of the European Parliament and of the Council Amending Regulation (EU) No 1093/2010 Establishing a European Supervisory Authority (European Banking Authority) (CON/2012/96), in: http://www.ecb.europa.eu/ecb/ legal/pdf/en_con_2012_96_f.pdf; 9.3.2013.

European Central Bank 2012c: Monthly Bulletin May 2012, in: http://www.ecb.europa.eu/pu b/pdf/other/art2_mb201205en_pp95-112en.pdf; 9.3.2013.

European Commission 2009: Beschluss der Kommission vom 23. Januar 2009 zur Einsetzung des Ausschusses der europäischen Bankaufsichtsbehörden (Text von Bedeutung für den EWR), in: http://eur-lex.europa.eu/LexUriServ/LexUriServ.do?uri=OJ:L:2009:025:0023 :01:DE:HTML; 9.3.2013.

European Commission 2010a: Proposal for a Directive.../.../EU of the European Parliament and of the Council on Deposit Guarantee Schemes [recast], in: http://ec.europa.eu/intern al_market/bank/docs/guarantee/20100712_proposal_en.pdf; 9.3.2013.

European Commission 2010b: Directive .../.../EU of the European Parliament and of the Council on Deposit Guarantee Schemes (COM(2010)368, Brüssel, 12.7.2010), in: http:// www.ipex.eu/IPEXL-WEB/dossier/files/download/082dbcc530b1bf490130bc5faf673; 9.3.2013.

European Commission 2011: Proposal for a Regulation of the European Parliament and the Council on the Strengthening of Economic and Budgetary Surveillance of Member States Experiencing or Threatened with Serious Difficulties with Respect to their Financial Stability in the Euro Area (COM(2011) 819, Brüssel, 23.11.2011), in: http://eur-lex.europa.eu/LexUriServ/LexUriServ.do?uri=COM:2011:0819:FIN:EN:PDF; 9.3.2013.

European Commission 2012a: Winding-up of Credit Institutions, in: http://ec.europa.eu/internal_market/bank/windingup/index_en.htm; 9.3.2013.

European Commission 2012b: The EU Single Market: Regulatory Capital, in: http://ec.europa.eu/internal_market/bank/regcapital/index_en.htm; 9.3.2013.

European Commission 2012c: Economic and Financial Affairs: EU Economic Governance, in: http://ec.europa.eu/economy_finance/economic_governance/index_en.htm; 9.3.2013.

European Commission 2012d: Lamfalussy Report, in: http://ec.europa.eu/internal_market/ securities/lamfalussy/index_en.htm; 9.3.2013.

European Commission 2012e: Proposal for a Council Regulation Conferring Specific Tasks on the European Central Bank Concerning Policies Relating to the Prudential Supervision of Credit Institutions (COM(2012) 511 final, Brüssel, 12.9.2012), in: http://eurlex.europa.eu/LexUriServ/LexUriServ.do?uri=COM:2012:0511:FIN:EN:PDF; 9.3.2013. 
European Commission 2012f: Proposal for a Regulation of the European Parliament and of the Council Amending Regulation (EU) No 1093/2010 Establishing a European Supervisory Authority (European Banking Authority) as Regards Its Interaction with Council Regulation (EU) No.../... Conferring Specific Tasks on the European Central Bank Concerning Policies Relating to the Prudential Supervision of Credit Institutions (COM(2012) 512 final, Brüssel, 12.9.2012), in: http://eur-lex.europa.eu/LexUriServ/ LexUriServ.do?uri=COM:2012:0512:FIN:EN:PDF; 9.3.2013.

European Council 2012a: European Council Conclusions on Completing EMU (Brüssel, 18 10.2012), in: http://www.consilium.europa.eu/uedocs/cms_data/docs/pressdata/en/ec/ 132986.pdf; 9.3.2013.

European Council 2012b: Vertrag über Stabilität, Koordinierung und Steuerung in der Wirtschafts- und Währungsunion, 2.3.2012, in: http://www.european-council.europa.eu/ media/639244/04_-_tscg.de.12.pdf; 9.3.2013.

Frankel, Jeffrey A./ Rose, Andrew K. 1996: The Endogeneity of the Optimum Currency Area Criteria (CEPR Discussion Papers 1473), London.

Grieco, Joseph M. 1995: State Interests and Institutional Rule Trajectories: A Neorealist Interpretation of The Maastricht Treaty and European Economic and Monetary Union, in: Security Studies 5: 3, 261-305.

Haas, Ernst 1958: The Uniting of Europe: Political, Social and Economic Forces, 1950-1957, London.

Haas, Ernst 1964: Technocracy, Pluralism and the New Europe, in: Graubard, Stephen (Hrsg.): A New Europe?, Boston, MA, 62-88.

Haas, Ernst 1970: The Study of Regional Integration: Reflections on the Joy and Anguish of Pretheorizing, in: International Organization 24, 607-644.

Huysmans, Jef 2000: The European Union and the Securitization of Migration, in: Journal of Common Market Studies 38: 5, 751-777.

Inter-Institutional Monitoring Group 2007: Final Report Monitoring the Lamfalussy Process, in: http://ec.europa.eu/internal_market/finances/docs/committees/071015_final_report_e n.pdf; 9.3.2013.

International Monetary Fund 2012: IMF and the Group of Twenty, in: http://www.imf.org/ external/np/g20/; 9.3.2013.

Jachtenfuchs, Markus 1997: Conceptualizing European Governance, in: Jorgensen, Knud E. (Hrsg.): Reflective Approaches to European Governance, Basingstoke.

Le Monde 2012: François Hollande: »L'Europe ne peut plus être en retard«, 17.10.2012, in: http://www.lemonde.fr/politique/article/2012/10/17/francois-hollande-l-europe-ne-peutplus-etre-en-retard_1776532_823448.html; 9.3.2013.

Liikanen, Erkki/Bänziger, Hugo/Campa, José Manuel/Gallois, Louis/Goyens, Monique/Krahnen, Jan Pieter/Mazzucchelli, Marco/Sergeant, Carol/Tuma, Zdenek/Vanhevel, Jan/ Wijffels, Herman 2012: High-level Expert Group on Reforming the Structure of the EU Banking Sector: Chaired by Erkki Liikanen: Final Report (Brüssel, 2.10.2012), in: http:// ec.europa.eu/internal_market/bank/docs/high-level_expert_group/report_en.pdf; 9.3.2013.

Lindberg, Leon 1963: The Political Dynamics of European Integration, Stanford, CA.

Merkel, Angela 2012a: Regierungserklärung von Bundeskanzlerin Merkel zu Finanzhilfen für Griechenland und Europäischer Rat am 1./2. März 2012 in Brüssel, 27.2.2012, in: http:// www.bundesregierung.de/Content/DE/Regierungserklaerung/2012/2012-02-27-merkel.h tml; 9.3.2013.

Merkel, Angela 2012b: Regierungserklärung von Bundeskanzlerin Merkel zum Europäischen Rat, Berlin, 18.10.2012.

Moravcsik, Andrew 1998: The Choice for Europe, Ithaca, NY.

Niemann, Arne 2006: Explaining Decisions in the European Union, Cambridge.

Niemann, Arne/Schmitter, Philippe 2009: Neo-Functionalism, in: Wiener, Antje/Diez, Thomas (Hrsg.): Theories of European Integration, 2. Auflage, Oxford, 45-66. 
Padoa-Schioppa, Tommaso 2000: The Road to Monetary Union in Europe: The Emperor, the Kings and the Genies, Oxford.

Pierson, Paul 1996: The Path to European Integration: A Historical Institutionalist Analysis, in: Comparative Political Studies 29: 2, 123-63.

Rajoy, Mariano 2012: Brief an Kommissionspräsident Barroso und Ratspräsident Van Rompuy, in: http://www.cnbc.com/id/47794623/Europe039s_039Worst_Crisis039_Requires_Fiscal_Union_Spain; 4.3.2013.

Risse, Thomas/Engelmann-Martin, Daniela/Knope, Hans/Roscher, Klaus 1999: To Euro or not to Euro: The EMU and Identity Politics in the European Union, in: European Journal of International Relations 5: 2, 147-187.

Sandholtz, Wayne 1993: Choosing Union: Monetary Politics and Maastricht, in: International Organization 47: 1, 1-39.

Siebert, Horst 1998: The Euro: A Dozen Do's and Don'ts, in: Kieler Diskussionsbeiträge 312.

Schimmelfennig, Frank 2012: Zwischen Neo- und Postfunktionalismus: Die Integrationstheorien und die Eurokrise, in: Politische Vierteljahresschrift 53: 4, 394-413.

Schoenmaker, Dirk 2011: The Financial Trilemma, in: Economics Letters 111: 1, 57-59.

Schoenmaker, Dirk 2012: Banking Supervision and Resolution: The European Dimension, January 2012, DSF Policy Paper No. 19.

Spiegel Online 2012: Krise in Spanien: »Harte Reformen sind nötig« (Interview mit Joaquín Almunia, 3.10.2012), in: http://www.spiegel.de/wirtschaft/soziales/eu-kommissar-almunia-zur-spanien-krise-machen-was-europa-rettet-a-859042.html; 10.3.2013.

The Guardian 2012: Eurozone Moves a Decisive Step Closer to Banking Union (Brüssel, 3.10.2012), in: http://www.guardian.co.uk/business/2012/dec/13/eurozone-banking-unio n-step-closer; 10.3.2013.

Tranholm-Mikkelsen, Jeppe 1991: Neo-functionalism: Obstinate or Obsolete? A Reappraisal in the Light of the New Dynamism of the EC, in: Millenium - Journal of International Studies 20: 1, 1-22.

Van Rompuy, Herman 2012a: Towards a Genuine Economic and Monetary Union: Report by President of the European Council Herman Van Rompuy (Brüssel, 26.6.2012), in: http:// www.consilium.europa.eu/uedocs/cms_data/docs/pressdata/en/ec/131201.pdf; 10.3.2013.

Van Rompuy, Herman 2012b: Towards a Genuine Economic and Monetary Union: Report by President of the European Council Herman Van Rompuy (Brüssel, 5.12.2012), in: http:// www.consilium.europa.eu/uedocs/cms_Data/docs/pressdata/en/ec/134069.pdf; 10.3.2013.

Van Rompuy, Herman/Barroso, José M./Juncker, Claude/Draghi, Mario 2012: Auf dem Weg zu einer echten Wirtschafts- und Währungsunion, in: http://www.consilium.europa.eu/ uedocs/cms_data/docs/pressdata/de/ec/134206.pdf; 10.3.2013.

Verdun, Amy 2007: A Historical Institutionalist Analysis of the Road to Economic and Monetary Union: A Journey with Many Crossroads, in: Meunier, Sophie/McNamara, Kathleen R. (Hrsg.): The State of the European Union: Making History: European Integration and Institutional Change at Fifty, Oxford, 197-209. 